\title{
Evolução dos Insetos-Praga na Cultura da Soja no Mato Grosso
}

\author{
Aleson Wiest¹ \& Marliton Rocha Barreto2 ${ }^{\bowtie}$
}

1. Universidade Federal de Mato Grosso, Instituto de Ciências Agrárias e Ambientais - ICAA, e-mail: aleson.wiest@fiagril.com.br. 2. Universidade Federal de Mato Grosso, Núcleo de Estudos da Biodiversidade da Amazônia Mato-grossense - NEBAM, e-mail: mrbarreto@ufmt.br (Autor para correspondência ${ }^{\varpi}$ ).

\section{EntomoBrasilis 5 (2): 84-87 (2012)}

Resumo. O presente trabalho apresenta o desenvolvimento da cultura da soja no estado de Mato Grosso quanto aos insetos-praga associados a essa cultura. Constatou-se que em 1978 essa cultura apresentava seis espécies-pragas principais e nenhuma praga secundária ou esporádica. No ano de 2008, 33 espécies são consideradas insetos-pragas nesta cultura, sendo 10 espécies consideradas pragas principais, 11 espécies secundárias e 12 espécies esporádicas. Esses dados demonstram um aumento superior a 450\% quanto à incidência de pragas da cultura da soja, no período 1978 2008, no estado do Mato Grosso, sendo que as pragas principais tiveram um aumento de 66,67\%.

Palavras-Chave: Insecta; Mato Grosso; Praga; Sojicultura.

\section{Evolution of Insect Pests in Soybeans in Mato Grosso}

Abstract. This study presents the development of soy crops in the state of Mato Grosso regarding the pest-insects that have been associated to that culture. It was found that in 1978 soybean crops had six main pest-species and no secondary or sporadic pests. In 2008 , 33 species were considered to be pest-insects to this culture, 10 of which are classified as main pests, 11 as secondary, and 12 as sporadic pests. These data show an increase of over $450 \%$ in the incidence of soybean crop pests from 1978 - 2008, in the state of Mato Grosso, with a 66.67\% increase in main pests.

Keywords: Insecta; Mato Grosso; Pest; Soybean Cultivation.

D e acordo com BERNo \& SCHNEIDER (2007) o espaço agrário do estado de Mato Grosso possuía grandes áreas de vegetação nativa de Cerrado e algumas áreas de pastagens naturais ou pastagens que ocorriam após o desmatamento das matas nesta região. Na década de 1970 já existiam, na região de Mato Grosso, algumas atividades agrícolas que eram praticadas em pequenas áreas por agricultores familiares.

Nos primeiros anos da década de 1970, o cultivo da soja passou a ser uma nova atividade econômica para o estado de Mato Grosso. Com a chegada da soja, seu cultivo foi mais elaborado, trazendo consigo novos conhecimentos e procedimentos, como a mecanização e a modernização das práticas de cultivo (BERNo \& SCHNEIDER 2007).

Com estímulos governamentais os grandes proprietários rurais da região sulista do país expandiram as fronteiras agrícolas com destino ao estado de Mato Grosso e com a chegada desses migrantes inicia-se uma reestruturação da paisagem natural do Centro-Oeste (DuARTE 1989). Os incentivos governamentais e os subsídios oferecidos para esses grandes produtores fizeram com que não só o espaço passa-se por mudanças, mas a própria agricultura.

Segundo Bonato (2000), as perdas de produtividade e da própria qualidade do grão da soja estão ocorrendo devido aos diversos fatores, que estão agindo sobre a cultura no decorrer do seu ciclo e um dos maiores problemas, que entravam o aumento de produtividade das plantas de soja é a ocorrência das "pragas", seguidos por doenças, nematóides e pela competição com as plantas daninhas (ChristofoletTI 2009).
De acordo com Cruz et al. (1995), à medida que o produtor rural no estado de Mato Grosso aumenta seu nível tecnológico e sua área de produção, o mesmo passa a utilizar um sistema mais intensivo de monocultura, e com isso normalmente, tem-se um aumento dos problemas entomológicos, pois o mesmo utiliza produtos químicos de maneira abusiva e inadequada, ocasionando resíduos dos produtos e a eliminação dos inimigos naturais, controlando apenas parcialmente a praga. Desta forma, o presente trabalho foi realizado de 2008 a 2010, através de pesquisas bibliográficas em artigos, dissertações, livros, teses, sites especializados, dentre outros e teve como objetivo diagnosticar as pragas da cultura da soja, no norte do estado de Mato Grosso, entre os anos de 1978 e 2008, possibilitando melhor conhecimento da entomofauna nesta cultura.

O conhecimento dos insetos que ocorrem numa cultura é muito importante para a programação de tratamentos fitossanitários e também para previsão do surgimento de novas pragas. De acordo com Silva (2000), uma das maiores preocupações encontradas pelos produtores de soja no estado de Mato Grosso é em relação aos insetos que causam danos diretos e indiretos a essa cultura, de acordo com o nível populacional, época de ocorrência e a parte da planta atacada, entre outros fatores. Quanto maior a extensão de área ocupada pela cultura da soja, maior será o número de insetos e de outros seres vivos que passam a se alimentar dessa cultura. No caso da soja, o número de insetos que se alimenta é superior a duas centenas, das quais apenas uma pequena parte causa prejuízos significativos a essa lavoura (SANTos 1988). Já GAZZONI \& MOSCARDI (1998) relataram que na cultura da soja, a maior parte dos insetos-praga, ocasiona danos indiretos, como redução da área foliar e o broqueamento de talos e ramos. 
Como a cultura da soja está sujeita ao ataque de insetos, praticamente, durante todo o seu ciclo, podemos definir esses ataques com as principais pragas em seus respectivos lugares desde início do plantio até a colheita. São consideradas pragas principais aquelas que ocorrem todos os anos, em altas populações, provocando danos econômicos para a cultura e por isso requer medidas de controle (EMBRAPA 2000). De acordo com GAZzon et al. (1981), pragas secundárias são aqueles insetos que possuem ampla distribuição geográfica, mas seus danos variam de região para região e, na maioria delas, os prejuízos causados não atingem expressão econômica, ou seja, essa praga aparece quando o produtor rural faz aplicação indiscriminada de agroquímicos eliminando assim as pragas principais e os inimigos naturais que existem nesta cultura, causando desequilíbrio na entomofauna da cultura da soja ou até mesmo por algumas condições edafoclimáticas que favorecem o surgimento das mesmas. A soja pode, também, ser atacada por outras espécies de insetos, considerados pragas esporádicas que, em geral, não ocorrem em todas as safras, ou os aumentos de população são determinados por alterações climáticas, ou outros fatores como, por exemplo, os sistemas de produção específicos de cada região (EMBRAPA 2000).

De acordo com Embrapa Algodão (2002), a abertura do cerrado para o cultivo da cultura da soja, fez com que a produção dessa cultura aumentasse significativamente a cada safra, isso se deu devido o aumento da área de produção. No entanto, esse crescimento agravou em alguns problemas como pragas e doenças que passaram a adquirir importâncias regionalizadas principalmente na região do estado de Mato Grosso. Com isso os insetos-pragas que só ocorriam em outros estados passaram a ocorrer no estado do Mato Grosso, ou seja, as pragas que atingiam a cultura da soja inicialmente eram consideradas esporádicas, mas começaram a atingí-las com mais freqüência. Pode-se ter como exemplo, o percevejo castanho-da-raiz (Scaptocoris castanea Perty), que possuía ocorrência esporádica associada à cultura da soja e, com a sua expansão para o Centro-Oeste, passou a se tornar um problema sério na cultura, que se agravou ainda mais a partir da década de 1990, quando a evolução do dano se tornou crescente, passando inclusive a ser constatado também em outras culturas, como o algodão.

Em função do cultivo da soja na região Centro-Oeste ter se tornado de larga escala, bem como devido ao aparecimento dessas pragas na cultura, os produtores sem experiência nenhuma passaram a utilizar insumos e defensivos sem controle algum e substituíram o sistema convencional pelo sistema de plantio direto, tentando assim dificultar a ação profilática desses organismos (EMBRAPA ALGODÃo 2002), que resultaram no desenvolvimento de novos conceitos e técnicas de controle de pragas tais como a fabricação de pulverizadores agrícolas (terrestres e aéreos) que começaram a fazer parte dessas enormes áreas cultivadas na região Central do Brasil (Christofoletti 2009).

No entanto, esses novos conceitos trouxeram consigo benefícios para essa cultura como à diminuição do tempo de intervenção das pragas na cultura da soja, auxiliando o produtor na sua tomada de decisão tanto no uso de defensivos preventivos como curativos no início das infestações, evitando assim grandes prejuízos econômicos. Contudo, o produtor rural tem a sua disposição, nos dias atuais, técnicas de aplicações de defensivos agrícolas seguras, mesmo em diferentes tipos de culturas, ambiente e ação de defensivo (ChristofoletTi 2009).

Quanto aos insetos-praga associados à cultura da soja, constatouse que em 1978 essa cultura apresentava seis espécies-pragas principais e nenhuma praga secundária ou esporádica de acordo com CANEquio Filho \& AlmeidA (1981). Mas, à medida que a cultura da soja ganhava espaço na região norte do estado de Mato Groso, devido ao aumento impulsivo de sua área plantada, de novas aberturas de terras e do uso abusivo de monocultura, houve um desequilíbrio na entomofauna da cultura da soja o que resultou no ano de 2008, na constatação de 10 espécies de insetos-praga principais, 11 espécies secundárias e 12 espécies consideradas pragas esporádicas (Tabela 1).

\section{Outras pragas}

Diversas pragas atacaram a cultura da soja no passado: Caliothrips brasiliensis Morgan, Chalcodermus sp., A. mourei, S. latifascia, Acrosternum sp., B. argentifolii, Blapstinus sp., Colaspis sp., E. atomaria, E. zinckenella, H. indicata, I. dives, Lagartas de Geometrídeos, $M$. testulalis, $M$. armatus, $P$. vittigera e $R$. nu (Tabela 1). Outra questão é que estas pragas não vêm mais causando prejuízos na cultura da soja no norte de Mato Grosso, ou seja, a partir do levantamento de 2008 as mesmas não foram nem enquadradas como pragas esporádicas da cultura da soja. Possivelmente, essas pragas foram erradicadas dessa cultura ou aparecem em números diminutos.

\section{Considerações Finais}

Esses dados demonstram um aumento superior a 500\% quanto à incidência de pragas da cultura da soja, nesses últimos 25 anos, no estado do Mato Grosso, sendo que as pragas principais tiveram um aumento de $66,67 \%$. Estatisticamente, as pragas secundárias tiveram um aumento de $\mathbf{1 . 1 0 0 \%}$ e as pragas esporádicas um aumento superior a $1.000 \%$.

Pode-se notar também que algumas pragas que atacavam a cultura da soja em 1978, hoje não mais causam danos. O que, em parte, pode ser atribuído a mudança de sistema de plantio adotada, na qual em 1978 utilizava-se muito o uso do sistema de plantio convencional e que passou alguns anos depois para o uso de sistema de plantio direto bem como pelo desenvolvimento de novos ingredientes ativos com diferentes sistemas de ação e seletividade, o que contribuiu para a manutenção dos inimigos naturais na área.

\section{REFERÊNCIAS}

Berno, M.L. \& C.I. Schneider, 2007. Modernização e mecanização agrícola em Palotina - PR: a idéia de desenvolvimento econômico e social no período de 1970 - 1983. Akrópólis, Umuarama, 15: 217-227.

Bonato, E. R., 2000. Estresses em soja. Passo Fundo: Embrapa Trigo. 254 p.

Borket, C. M., T. J. Yorinori, B.S. Corrêa-Ferreira, A.M.R. Almeida, L.P. Ferreira \& G.J. Sfredo, 1994. Seja o doutor da sua soja. Informe Agronômico, 6:1-66.

Canequio Filho, V. \& T.C. Almeida, 1981. Principais culturas. Instituto Campineiro de Ensino Agrícola. Vol II. 407 p.

Christofoletti, J.C., 2009. A produção de alimentos. Disponível em: <http://www.ctnbio.gov.br/upd blob/0001/1058.pdf $>$. Acesso em 25 Mar. 2009.

Cruz, I., J.M. Waquil, P.A. Viana \& F.H. Valicente, 1995. Pragas: Diagnóstico e controle. In Arquivo do Agrônomo. Seja o doutor do seu milho. Potafós, São Paulo. 2: 9-21.

Degrande, P.E. \& L.M. Vivan, 2008. Pragas da soja. In: FUNDAÇÃO MT. Boletim de pesquisa de soja 2008. Rondonópolis, p. 123-160.

Duarte, A.C. 1989. O Centro-Oeste na organização regional do Brasil. In: BRASIL. IBGE. Geografia do Brasil: região CentroOeste. Rio de Janeiro: IBGE, p. 15-20.

Embrapa Algodão, 2002. Complexo de Percevejos da Soja e Percevejo-Castanho-da-raiz: Problemas Ocasionados à Cultura do Algodão. Campina Grande, 28p. (Embrapa Algodão. Documentos, 103).

Embrapa. 2000. Recomendações técnicas para a cultura da soja na Região central do Brasil. 2000/2001. Cuiabá 245p. (Embrapa Soja/ Fundação - MT Documentos, 146).

Gazzoni, D.L. \& F. Moscardi, 1998. Effect of defoliation levels on recovery of leaf area, on yield and agronomic traits of soybeans. Pesquisa Agropecuária Brasileira. Brasília, 33: 411424. 


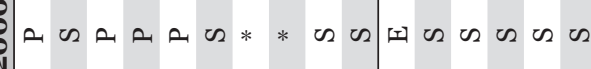

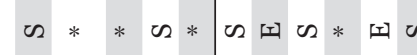

ริ

$\infty$

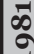

$\infty$

20

$\ln a \sin \theta \cos$

$\div$

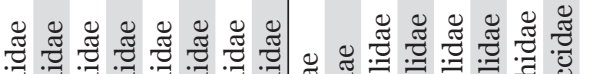

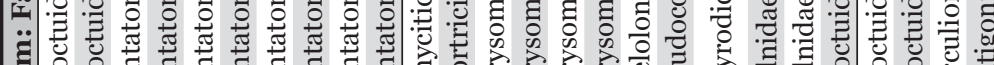

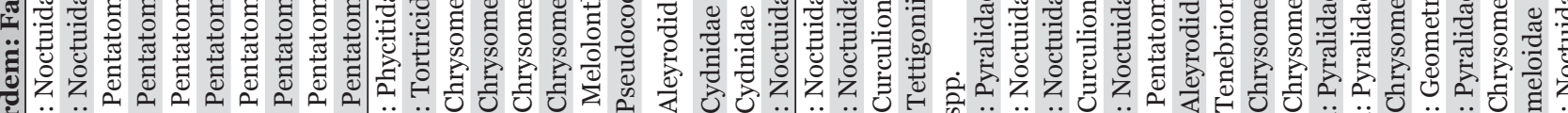

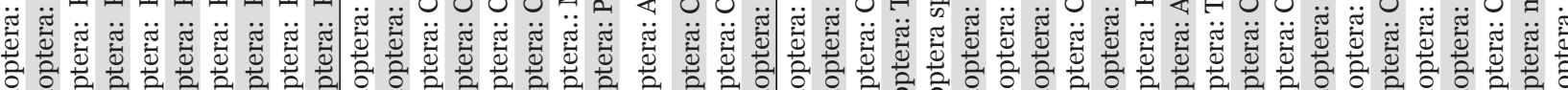

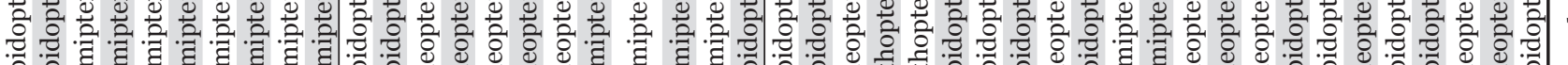

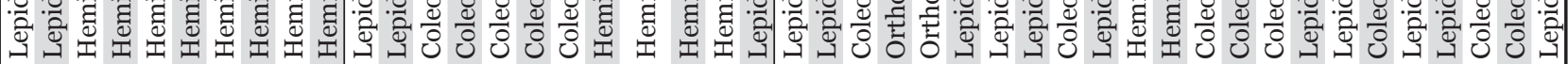

足

$\frac{\pi}{\circ}$

है

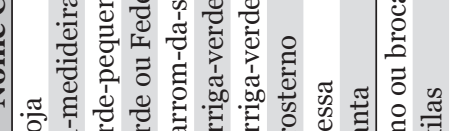

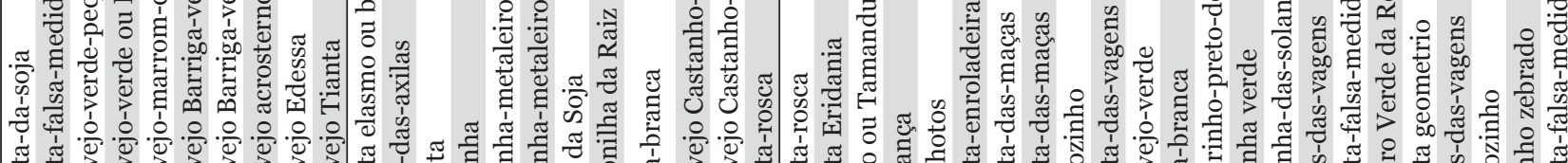

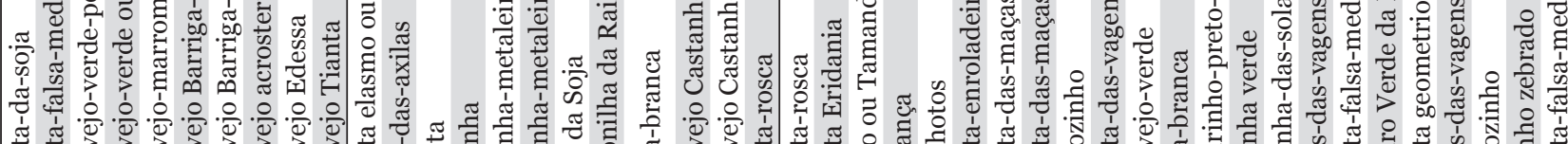

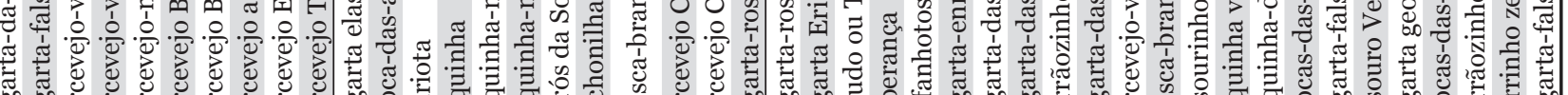

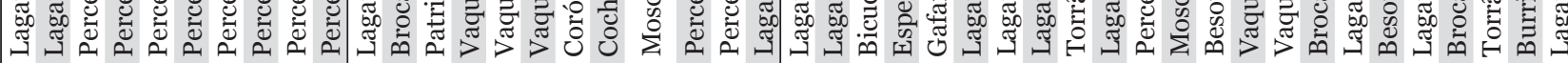

8

馬苛 
Gazzoni, D.L., E.B. Oliveira, I.C. Corso, B.S. Corrêa-Ferreira, G.L. Villas Boas, F. Moscardi \& A.R. Panizzi, 1981. Manejo de pragas da soja. Londrina: EMBRAPA - CNPSo, 44p. (Embrapa - CNPSo. Circular Técnica, 5).

Hoffmann-Campo, C.B., F. Moscardi, B.S. Corrêa-Ferreira, L.J. Oliveira, D.R Sosa-Gomez, A.R. Panizzi, I.C. Corso, D.L. Gazzoni, \& E.B. Oliveira, 200o. Pragas da soja no Brasil e seu manejo integrado. Londrina: EMBRAPA SOJA. 70p. (Circular Técnica, 30).

Miyasaka, S. \& J.C. Medina, 1981. A soja no Brasil. ITAL: Campinas, 1062p.

Santos, O.S., 1988, A cultura da soja, 1: Rio Grande do Sul, Santa Catarina, Paraná / O. S. Santos (coord.) - Rio de Janeiro:
Globo, (Coleção do agricultor, Grãos) (Publicações Globo Rural).

Silva, M.T.B, 200o. Manejo de insetos nas culturas de milho e soja. In: Guedes, J.V.C., I.D. Costa \& E. Castiglion (Eds.). Bases e técnicas do manejo de insetos. Santa Maria: UFSM/ CCR/DFS, 248p.

\section{Recebido em: o8/o7/2011}

Aceito em: 25/09/2011

Como citar este artigo:

Wiest, A. \& M.R. Barreto, 2012. Evolução dos Insetos-Praga na Cultura da Soja no Mato Grosso. EntomoBrasilis, 5(2): 84-87.

Acessível em: http://www.periodico.ebras.bio.br/ojs/index.php/ebras/article/view/173
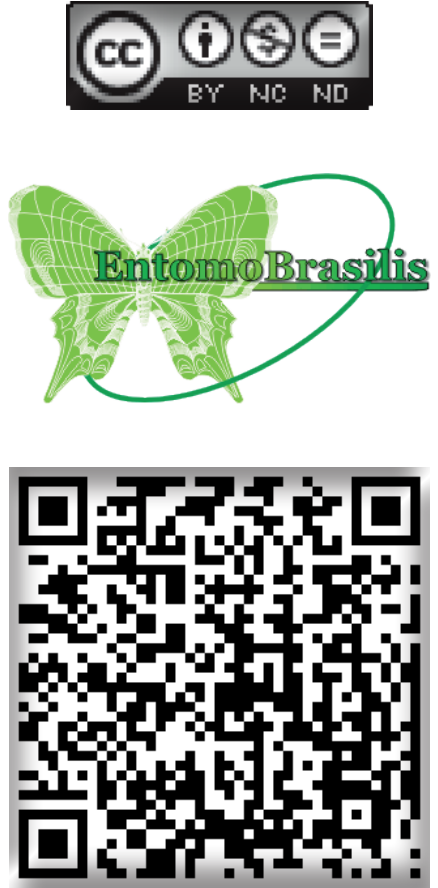